\title{
HABILIDADES SOCIALES EN ENTORNOS VIRTUALES DE TRABAJO COLABORATIVO
}

\author{
(SOCIAL SKILLS IN COLABORATIVE WORK VIRTUAL ENVIRONMENTS)
}

\author{
Nallilda Villasana \\ Elena Dorrego \\ Universidad Central de Venezuela (Venezuela)
}

\section{RESUMEN}

Este estudio se incluye dentro de la temática de investigación de las Tecnologías de la Información y la Comunicación, el diseño e implementación de metodologías de aprendizaje colaborativo y la promoción de habilidades sociales en estudiantes de nivel superior. El estudio fue de tipo exploratorio y se inserta dentro de los propósitos de la investigación preexperimental. Se administró un tratamiento al grupo y se midieron varias variables para observar el aprendizaje logrado a partir de la aplicación de una metodología de trabajo colaborativo basado en el Aprendizaje por Proyectos, la opinión de los estudiantes sobre la importancia del trabajo colaborativo y las habilidades sociales que evidenciaron a través del entorno virtual utilizado. Los resultados mostraron que una metodología de trabajo colaborativo basada en el aprendizaje por proyectos y mediada a través de un entorno virtual, promueve el desarrollo de habilidades sociales de conversación, y de aceptación y oposición asertiva en los estudiantes.

Palabras clave: entornos virtuales de enseñanza y de aprendizaje, trabajo colaborativo, habilidades sociales conversacionales y asertivas.

\begin{abstract}
This study is enclosed into the Information and Communication Technologies research, the design and implementation of collaborative learning methodologies and the promotion of social skills in higher education students. It was an exploratory study, and a preexperimental research. A treatment was applied to the group, and variables were assessed to observe the learning achieved from the application of a collaborative work methodology based on the learning by projects, the students' opinion about the importance of the collaborative work and social skills showed in the learning environment used. Results made evident that a collaborative work methodology based on learning by projects and mediated in a
\end{abstract}


virtual environment, promote the development of conversation, and assertive acceptance and opposition skills in students.

Key words: teaching and learning virtual environments, collaborative work, conversational and assertive social skills.

El uso cada vez más frecuente de las Tecnologías de la Información y la Comunicación (TIC), se ha hecho presente en el ámbito de la educación, sobre todo en el nivel de educación superior, donde el campo de la investigación en esta área día a día cuenta con más seguidores.

Ejemplo de lo anterior lo constituye este trabajo, el cual presenta una experiencia pedagógica que se enmarca dentro de la temática de investigación de las TIC, el diseño e implementación de metodologías de aprendizaje colaborativo y la promoción de habilidades sociales en estudiantes de nivel superior.

Uno de los aspectos más importantes que contribuyen al mejor desenvolvimiento en la sociedad lo constituyen las habilidades sociales que el individuo demuestre en el entorno donde se desarrolla. En el ámbito educativo, con frecuencia estas habilidades no son consideradas significativas a la hora de diseñar e implementar los procesos de enseñanza y de aprendizaje, sin embargo éstas componen elementos esenciales que deben ser tomados en cuenta para garantizar procesos efectivos que además coadyuven al crecimiento personal de los estudiantes.

En diversas investigaciones se evidencia que existe gran interés por conocer si la aplicación de métodos, técnicas o estrategias de trabajo colaborativo utilizando diferentes herramientas tecnológicas, facilita el aprendizaje en los alumnos, sin embargo, parecen escasas las que apuntan su interés hacia la investigación de las ventajas que pudiesen aportar las mismas en el desarrollo de habilidades sociales, aún cuando algunos autores han descrito la importancia de estas habilidades sobre todo en los nuevos entornos a los que debemos adaptarnos. Esta situación da lugar a la necesidad de diseñar estrategias instruccionales basadas en métodos de trabajo colaborativo, que garanticen no sólo la efectividad tanto del aprendizaje como del medio, sino también el desarrollo de habilidades sociales.

En este sentido, el propósito de la presente investigación fue indagar sobre las ventajas que pudiera aportar el trabajo colaborativo en entornos virtuales para promover el desarrollo de habilidades sociales. Se tomaron como variables las habilidades específicas de conversación y de asertividad porque sus características 
resultan las más adecuadas para la población objeto de estudio, y se observaron tomando en cuenta las interrelaciones personales que se produjeron entre los estudiantes.

Como eje central de esta investigación, se hizo especial énfasis en las habilidades sociales en el trabajo colaborativo, sus definiciones, tipos, estrategias para evaluarlas o medirlas, los inventarios más utilizados y experiencias del desarrollo de estas habilidades en entornos virtuales. Igualmente, se trató el tema sobre las TIC y los entornos de trabajo colaborativo, sus definiciones y las herramientas tecnológicas para trabajar con esta metodología.

Los resultados de la investigación permitieron comprobar que aplicar una metodología de trabajo colaborativo basada en el Aprendizaje por Proyectos y mediada a través de un entorno virtual en el contexto universitario, promueven el desarrollo de habilidades sociales de conversación y de aceptación y oposición asertiva en los estudiantes.

\section{UNA APROXIMACIÓN AL TRABAJO COLABORATIVO}

La literatura revisada mostró que los términos "cooperación" y "colaboración" se utilizan indistintamente, es decir, las definiciones de estos conceptos son muy similares aunque para algunos especialistas existen diferencias conceptuales y operativas entre los mismos.

Al respecto, Slavin (1993) citado por Alfageme (2002, p. 84), considera que “... con frecuencia, ambas tradiciones de investigación se enfrentan, aunque en realidad son complementarias. Los estudios sobre el aprendizaje cooperativo contribuyen a definir una estructura de motivación y de organización para un programa global de trabajo en grupo, mientras los estudios sobre el aprendizaje colaborativo se centran en las ventajas cognitivas derivadas de los intercambios más íntimos que tienen lugar al trabajar juntos".

Para Serrano y Calvo (1994) citados por Alfageme (2002, p. 114), “... el trabajo colaborativo no se orienta exclusivamente hacia el producto de tipo académico, sino que también persigue una mejora de las propias relaciones sociales. En este caso se considera esencial analizar la interacción producida entre el profesor y el alumno, pero también la interacción alumno-alumno". 
Panitz (1997), citado por Zañartu (2003, p. 2) señala que “...la premisa básica del aprendizaje colaborativo es la construcción del consenso, a través de la cooperación de los miembros del grupo". Señala que en el aprendizaje colaborativo se comparte la autoridad y entre todos se acepta la responsabilidad de las acciones del grupo; mientras que en la cooperación la interacción está diseñada para facilitar el logro de una meta o producto final específico por un grupo de personas que trabajan juntos.

Para Cabero (2003, p. 135), puede considerarse como “...una metodología de enseñanza basada en la creencia de que el aprendizaje se incrementa cuando los estudiantes desarrollan destrezas cooperativas para aprender y solucionar los problemas y acciones educativas en las cuales se ven inmersos".

Por su parte, Prendes (2003, p. 102) señala que el trabajo colaborativo es “... un método de enseñanza que basado en el trabajo grupal persigue una mejora del rendimiento y de la interacción entre los alumnos".

Los distintos conceptos se consideraron como aportes teóricos para integrar una definición del trabajo colaborativo asumiéndolo como un método de enseñanza adaptable a cualquier modalidad educativa, que a través del trabajo en grupo para alcanzar objetivos comunes utilizando diferentes técnicas o estrategias, busca mejorar la adquisición y desarrollo de habilidades cognitivas o intelectuales y habilidades sociales, mediante la interacción, la interdependencia y las relaciones interpersonales que se produzcan entre los grupos, durante y después de la aplicación del método.

El trabajo colaborativo, según diferentes autores, presenta una serie de características particulares que lo diferencian del trabajo en grupo tradicional. (Driscoll y Vergara, 1997, citados por Zañartu, 2003; Johnson, Johnson y Holubec, 1999, citados por Alfageme, 2002; Prendes, 2003; Román, 2002).

En esta investigación se procuró tener presentes características comunes atribuidas al aprendizaje colaborativo, al diseñar la tarea eimplementar la experiencia, tratando de establecer una cultura de colaboración entre los alumnos y docentes, incentivando la responsabilidad individual y la participación de los alumnos en la consecución de los proyectos, el intercambio de roles, la autoevaluación de sus acciones, entre otros, que finalmente permitieron obtener el mayor éxito posible. 


\section{HABILIDADES SOCIALES}

En este trabajo se tomaron como referencias las definiciones aportadas por tres autores, quienes dan diferentes matices acerca de un tema complejo para precisar con exactitud, por lo que han decidido aproximarse a lo que pudiese llamarse una "conducta socialmente habilidosa" (Alfageme, 2002, pp. 258-260), ha recopilado diferentes definiciones aportadas por algunos autores.

Caballo (1993), citado por Alfageme (2002, pp. 258-260) afirma que, “... la conducta socialmente habilidosa es ese conjunto de conductas emitidas por un individuo en un contexto interpersonal que expresa los sentimientos, actitudes, deseos, opiniones o derechos de ese individuo de un modo adecuado a la situación, respetando esas conductas en los demás, y que generalmente resuelve los problemas inmediatos de la situación mientras minimiza la probabilidad de futuros problemas". En opinión de este autor, "...definir una conducta habilidosa implica especificar tres componentes o dimensiones de la habilidad social: una dimensión conductual (tipo de habilidad), una dimensión personal (las variables cognitivas), y una dimensión situacional (el contexto ambiental)".

Para Calleja (1997), citado por Alfageme (2002, pp. 258-260), “...la conducta social implica la existencia de dos dimensiones principales: variables de la persona y variables de la situación. De modo que entre ellas puede establecerse que a mayor estructuración de la situación se requiere una menor competencia social; y a la inversa, a menor estructuración de la situación, la persona precisa de mayor competencia".

Por su parte, Kelly (1992, p. 19) define las habilidades sociales como “...aquellas conductas aprendidas que ponen en juego las personas en situaciones interpersonales para obtener o mantener reforzamiento del ambiente. Entendidas de esta manera, las habilidades sociales pueden considerarse como vías o rutas hacia los objetivos de un individuo".

Entre las habilidades sociales más significativas mencionadas con frecuencia por algunos autores, se encuentran las heterosociales o de iniciación de citas, las conversacionales, las de asertividad, las habilidades cooperativas, para entrevistas de trabajo, de juego cooperativo en niños, entre otras.

Considerando las características de esta investigación, en lo que se refiere a la aplicación de una metodología de trabajo colaborativo a través de un entorno virtual, 
se seleccionaron como variables las habilidades de conversación y de asertividad (aceptación asertiva y oposición asertiva), debido a que por sus características resultan las habilidades sociales básicas adecuadas para la población objeto de estudio.

En este sentido, Kelly (1992, p. 139) define las habilidades conversacionales como "... la capacidad de iniciar y mantener conversaciones informales con otras personas. Un repertorio conversacional efectivo no sólo permite al individuo salir airoso en las interacciones informales, sino que además es un precursor probable del desarrollo de relaciones duraderas, dado que la formación de tales relaciones requiere, en primer lugar, que el individuo interactúe satisfactoriamente con los demás durante una conversación. A diferencia de otros tipos de habilidades sociales que sirven para propósitos relativamente limitados, la competencia conversacional es necesaria en un amplio abanico de situaciones interpersonales cotidianas".

El mismo autor opina que hay que tener en cuenta que la competencia o la facilidad de interacción conversacional que tiene el sujeto depende de factores situacionales, incluida la familiaridad con el otro participante en la conversación, el sexo del cliente, el sexo del interlocutor y el propósito de la interacción.

Los componentes conductuales generales relacionados con la habilidad conversacional son, el contacto visual, el afecto, las preguntas conversacionales, los comentarios autorreveladores y los componentes de refuerzo o cumplidos. Algunos de estos componentes se tomaron como indicadores para medir la variable sobre habilidades conversacionales, otros fueron adaptados de acuerdo a las características del medio y a la modalidad de estudio implementada en la investigación.

En lo que se refiere a las habilidades de asertividad, Kelly (1992, p. 175) las define como "...la capacidad de un individuo para trasmitir a otra persona sus posturas, opiniones, creencias o sentimientos de manera eficaz y sin sentirse incómodo”.

Según los autores antes mencionados, el desempeño de la habilidad de asertividad puede dar lugar a la aceptación asertiva y a la oposición asertiva. La primera, se refiere a la habilidad de un individuo para transmitir calidez y expresar cumplidos u opiniones a los demás cuando la conducta positiva de éstos lo justifica. Los componentes de este tipo de habilidad, son los siguientes: el afecto, las expresiones de elogio/aprecio, la expresión de sentimientos personales y la conducta positiva recíproca. 
La oposición asertiva difiere de las formas de competencia social en cuanto a que no va dirigida principalmente a establecer nuevas relaciones sociales, más bien sirve para impedir la pérdida de reforzamiento, es decir, el individuo se opone o rechaza la conducta inaceptable del antagonista y trata de conseguir una conducta más aceptable en el futuro. En resumen, plantea Kelly (1992, p. 176) “...el objetivo de la oposición asertiva es comunicar tranquilamente nuestros sentimientos e invitar al antagonista a que cambie su conducta; no es descargar comentarios beligerantes sobre el otro". Los componentes conductuales de esta habilidad son el contacto visual, afecto y volumen de la voz, la comprensión de lo que el otro dice o expresión del problema, el desacuerdo y la petición de un cambio de conducta o propuesta de solución.

Para medir ambas variables de aceptación y oposición asertiva también se tomaron como indicadores algunos de estos componentes conductuales, otros se adaptaron considerando las características de la investigación.

\section{ENTORNOS VIRTUALES DE TRABAJO COLABORATIVO}

El avance de las TIC en el ámbito educativo ha suscitado el surgimiento de lo que hoy conocemos como entornos virtuales de enseñanza y de aprendizaje que, aprovechando las potencialidades de las TIC, ofrecen nuevos espacios para la enseñanza y el aprendizaje libres de las restricciones que imponen el tiempo y el espacio en la enseñanza presencial y capaces de asegurar una continua comunicación entre estudiantes y profesores. Estos entornos hoy en día utilizados en diferentes instituciones, también permiten complementar la enseñanza presencial con actividades virtuales, es decir, utilizarlas como apoyo al proceso instruccional.

Estableciendo una diferenciación entre los entornos de formación presencial y los virtuales, Gisbert, Adell, Rallo, y Bellver (1997, p. 113) señalan que “...en general, cuando se hace referencia a los entornos presenciales de formación se piensa en un espacio cerrado (un aula en una institución educativa) y utilizando los materiales habituales (libros, blocs de notas, mesas, sillas, etc.) y con procesos de comunicación cara a cara. Al contrario cuando se habla de entornos virtuales de formación, se deben tomar necesariamente otros referentes, por ejemplo un espacio de comunicación que integra un extenso grupo de materiales y recursos diseñados y desarrollados para facilitar y optimizar el proceso de aprendizaje de los alumnos y basado en técnicas de comunicación mediadas por el computador". 
$\mathrm{Al}$ respecto, algunos autores han tratado de definir el concepto de entornos virtuales de aprendizaje (EVA), entre ellos Barajas (2003) considera que “...son dominios en línea que permiten la interacción síncrona y asíncrona entre el profesorado y el alumnado. Además, contienen recursos de aprendizaje que pueden utilizarse por los estudiantes en cualquier momento".

Por su parte, Dillenbourg (1998) citado por Cebrián (2003, p. 154), señala que un entorno de enseñanza virtual:

- $\quad$ Es un espacio diseñado, no una mera acumulación de páginas HTML; es una arquitectura fruto del análisis de los requerimientos, capaz de evolucionar técnicamente y con una autoría múltiple: profesores, alumnos, expertos;

- Es un espacio social, un marco para el comportamiento interactivo;

- Ofrece una representación explícita que, más allá de que sea un interfaz textual o una compleja realidad virtual en $3 \mathrm{D}$, ejerce un efecto en el comportamiento de los usuarios;

- Permite que los alumnos sean productores de la información, proporcionando una experiencia más rica que el aprendizaje individual;

- No está restringido a la educación a distancia tradicional sino que puede complementar la educación presencial;

- Integra múltiples herramientas.

A partir de las reflexiones anteriores, puede decirse que los entornos virtuales se caracterizan por ampliar el acceso a la educación, promover el aprendizaje y el trabajo en grupo, promover el aprendizaje activo, crear comunidades de aprendizaje, estar centrada en el estudiante y hacer los roles tradicionales del proceso de enseñanza/ aprendizaje más fluidos.

Los elementos puramente tecnológicos quedan, por tanto, al margen de la definición de lo que debe ser un entorno integrado de enseñanza virtual que, por el contrario, se caracteriza por su capacidad para constituirse como elemento de innovación educativa, por la prioridad de la actividad sobre los contenidos, la participación creativa de los alumnos y el trabajo en colaboración. 


\section{METODOLOGÍA}

De acuerdo con el problema y los objetivos propuestos, se realizó un estudio de tipo exploratorio, debido a que los hallazgos encontrados sobre el tema evidenciaron escasas investigaciones relacionadas con las ventajas que puede aportar el trabajo colaborativo en un entorno virtual para el desarrollo de habilidades sociales (conversacionales y asertivas), en alumnos del contexto universitario.

Atendiendo a sus objetivos, la investigación se orientó hacia un diseño de campo, que permitió observar y recolectar los datos directamente de la realidad objeto de estudio, en su ambiente cotidiano, para posteriormente analizar e interpretar los resultados de las indagaciones. El seguimiento del trabajo de campo se realizó durante la elaboración de los proyectos por parte de diferentes grupos de alumnos, quienes durante un tiempo determinado participaron en diversas actividades a distancia utilizando un método de trabajo colaborativo y con el apoyo tecnológico de la Plataforma Synergeia, entorno virtual que facilitó un informe diario de las actividades realizadas en su espacio.

El estudio se inserta dentro de los propósitos de la investigación pre-experimental (Hernández, Fernández y Baptista, 1998). Se administró un tratamiento al grupo y luego se midieron varias variables para observar en qué nivel se ubicó el grupo con relación a: el aprendizaje logrado a partir de la aplicación de una metodología de trabajo colaborativo basado en el Aprendizaje por Proyectos, la opinión de los estudiantes sobre la importancia del trabajo colaborativo y las habilidades de conversación y de asertividad (aceptación asertiva y oposición asertiva) que se evidenciaron a través del entorno virtual utilizado.

La población estuvo constituida por los estudiantes de la Maestría en Educación, Mención Tecnologías de la Información y la Comunicación de la UCV, quienes cuentan con el perfil requerido para el estudio; la muestra la conformaron inicialmente los 23 estudiantes de la asignatura Modalidades Educativas y Tecnologías y finalmente por deserción de un estudiante y de dos, quedó conformada por 20 sujetos. La muestra se define como no probabilística, por ser la que más se ajusta a los objetivos del estudio, al diseño de la investigación y a la contribución que pretende aportar, por tratarse de un estudio exploratorio que intenta documentar una experiencia y generar datos que sirvan de base para investigaciones posteriores.

Los instrumentos utilizados para la recolección de la información fueron los siguientes: 
- “Cuestionario de opinión sobre habilidades intelectuales y sociales", aplicado al comienzo de la experiencia, para solicitar a los alumnos algunos datos sobre la conformación de los grupos.

- $\quad$ Para establecer el logro del aprendizaje basado en proyectos alcanzado por los alumnos de acuerdo con los contenidos trabajados en la experiencia, se evaluó cada grupo con ayuda de dos instrumentos: una "Hoja de Registro" para medir la calidad del trabajo realizado y un "Registro de las Participaciones", para determinar el nivel y calidad de las intervenciones y aportes de cada alumno.

- "Cuestionario de Percepción sobre el Trabajo Colaborativo", para medir el nivel de conocimientos y percepción de los alumnos sobre la importancia de este tipo de metodología.

- Para las habilidades conversacionales y de aceptación y oposición asertiva evidenciadas por los estudiantes, se analizaron cada una de las participaciones clasificándolas de acuerdo al propósito de las mismas, a las dimensiones de cada variable y a las categorías establecidas, la información se recolectó utilizando "Hojas de Registro".

Este conjunto de instrumentos, permitió hacer una evaluación general de las ventajas que aporta el trabajo colaborativo en un entorno virtual para promover habilidades conversacionales y asertivas, así mismo permitió conocer el nivel de conocimiento de los alumnos en cuanto al tema, y sus percepciones sobre la importancia del trabajo colaborativo.

\section{Descripción de la metodología}

Fase 1. Revisión documental. Constituyó una de las partes más importantes de este proceso investigativo, pues no sólo brindó información oportuna, sino también, permitió confrontar ideas, complementar planteamientos, confirmar criterios, ampliar visiones y definir reflexiones acerca del tema objeto de estudio. Abarcó la indagación en fuentes documentales primarias y secundarias impresas y en línea a fin de ubicar y seleccionar el constructo teórico base de la investigación, algunas experiencias relacionadas con el tema objeto de estudio; y la exploración de distintas herramientas de trabajo colaborativo, a fin de seleccionar la más apropiada para el desarrollo de la experiencia.

Fase 2. Organización y desarrollo del proyecto. Comprendió todo el conjunto de acciones llevadas a cabo antes y durante la experiencia, las cuales se describen a continuación. 
- Contacto con los alumnos. Se informó a los alumnos sobre la realización de la investigación, y se solicitó su consentimiento para participar, el cual se logró totalmente.

- Selección de la plataforma de trabajo. Se seleccionó para la implementación de la experiencia la Plataforma de Trabajo Colaborativo "Synergeia", entre otras razones, porque:

- Es una herramienta de Aprendizaje Colaborativo Soportado por Computadora (CSCL), que apoya la funcionalidad de un grupo colaborativo y permite la construcción compartida del conocimiento y la propiedad del grupo.

- Su diseño es guiado por un alcance educacional que enfatiza la construcción del conocimiento dentro de una comunidad de aprendices.

- $\quad$ La construcción del conocimiento resulta mayormente de la discusión personal, grupal y perspectiva del curso.

- $\quad$ La interfaz de usuario de las áreas de discusión hiladas en Synergeia han sido diseñadas para estimular la construcción del conocimiento de manera concentrada, pensada y profunda.

- Es de fácil acceso y el servidor BSCW en el que se encuentra alojada, puede ser usado de forma gratuita por todas las personas interesadas.

Finalizado el trabajo en este entorno se constató a través de las participaciones e interacciones de los alumnos que es una herramienta de fácil manejo, con una interfaz de navegación muy sencilla, y que además ofrece amplias posibilidades para el trabajo en grupo.

- Aplicación del Cuestionario de Opinión sobre habilidades intelectuales y sociales. Antes de iniciar la actividad en Synergeia, se aplicó este cuestionario a los alumnos, con la finalidad de obtener información sobre la opinión que tenían acerca de cada uno de sus compañeros, a partir de una experiencia de trabajo en grupo previamente realizada durante la asignatura.

Para ello, debieron indicar su opinión considerando las categorías Alta, Media o Baja en cuanto a las habilidades cognitivas y sociales de cada uno de sus compañeros. Este cuestionario tuvo un carácter confidencial, y su utilidad sólo se suscribió a arrojar información requerida para la conformación de grupos heterogéneos en cuanto a habilidades cognitivas y sociales. 
- Suscripción de los alumnos. Previamente el grupo de alumnos fue informado sobre la plataforma de trabajo colaborativo seleccionada como soporte para desarrollar la experiencia. Una vez creado el grupo, se invitó a todos a suscribirse en Synergeia. La primera semana consistió en la familiarización con esta herramienta, durante la misma se verificó la suscripción de cada uno y una vez comprobada, se dio por iniciada la experiencia. Adicionalmente se facilitó de forma presencial una breve inducción sobre el uso y las potencialidades educativas que ofrece la plataforma.

- Distribución de los grupos. Una vez suscritos todos los estudiantes en Synergeia, se les informó la distribución de los grupos de trabajo, la misma se realizó atendiendo al grado de heterogeneidad que se propone en la metodología de trabajo colaborativo en cuanto al nivel de habilidades cognitivas y sociales que deben concertarse en un grupo para garantizar su éxito. La información que arrojó la aplicación del "Cuestionario de Opinión sobre habilidades intelectuales y sociales" sirvió para organizar los grupos de manera heterogénea.

Dentro del entorno se abrió un espacio para cada uno de estos grupos, creando así cuatro subgrupos de trabajo, cada uno de los cuales tuvo la posibilidad de organizar su espacio según los requerimientos de las tareas asignadas y las posibilidades que ofrece Synergeia, entre ellas: crear carpetas y subcarpetas, colocar documentos, comunicarse asincrónicamente a través de la creación de foros y debates, colocar URLs y utilizar la comunicación sincrónica utilizando la herramienta MAPTOOL.

- Propuesta de roles. Atendiendo a la metodología de trabajo colaborativo en cuanto al desempeño e intercambio de roles dentro del proceso instruccional, luego de revisar varias posturas se adoptaron y sugirieron a los estudiantes, los siguientes roles:

- $\quad$ el iniciador y contribuidor, quien sugiere o propone al grupo nuevas ideas o formas de considerar los problemas o los objetivos del grupo.

- $\quad$ el que busca información, quien deberá no sólo solicitar la aclaración de las sugerencias, sino pedir información autorizada y los hechos pertinentes al problema en discusión.

- $\quad$ el compendiador, quien reúne las ideas y comentarios de los integrantes del grupo, así como las decisiones para ayudar a determinar dónde está el grupo en su proceso. 
- $\quad$ el integrador y coordinador, quien se encarga de aclarar las relaciones entre las diversas ideas, extrayendo las ideas clave de las contribuciones de los miembros e integrándolas en un todo significativo.

- $\quad$ el estimulador, quien incita al grupo a una acción o a una decisión, intenta estimularlo hacia una mayor o mejor actividad.

- el que está en desacuerdo, cuya labor tiene que ser argumentar en contra, denotar errores en los hechos o en los razonamientos.

- Aspectos considerados para desarrollar el Proyecto. Con la finalidad de que las actividades propuestas en esta investigación para el desarrollo del proyecto, se llevaran a cabo de una manera organizada y acorde con lo que significa este método de trabajo, se asumió la metodología basada en el Aprendizaje por Proyectos, la cual propone la resolución de tareas construidas sobre un trabajo previo elaborado, que en este caso se realizó a través de la puesta en práctica del modelo de trabajo colaborativo de investigación en grupo (Group Investigation) de Herbert Thelen, completado por Sharan, (Román, 2002), cuyos pasos a seguir son los siguientes:

- $\quad$ Selección del tópico.

- $\quad$ Planeación cooperativa de metas, tareas y procedimientos.

- Implementación: despliegue de una variedad de habilidades y actividades; monitoreo del profesor.

- $\quad$ Análisis y síntesis de lo trabajado y del proceso seguido.

- Presentación del producto final.

- Evaluación.

Bajo esta visión y acorde con el contenido a trabajar, "Los Entornos Virtuales de Enseñanza y de Aprendizaje en la Educación Superior. Un caso venezolano”, y el objetivo general del proyecto, el cual se orientó hacia "Caracterizar los entornos virtuales de enseñanza y de aprendizaje en la educación superior, aplicado a un caso venezolano se organizó el trabajo en tres momentos:

- Planificación de la metodología de trabajo. Esta actividad se centró en que los estudiantes planificaran la metodología de trabajo a llevar a cabo para elaborar el proyecto. Además de sugerirles los aspectos claves para realizarla, se abrió un foro, como un espacio para consultar las dudas acerca de las actividades a realizar. Se observó que cada subgrupo se organizó de manera 
asertiva, construyendo espacios para colocar los avances que serían revisados por los integrantes de cada grupo, foros internos de discusión sobre las tareas a realizar, debates sobre algunos puntos, carpetas para colocar los trabajos finales, entre otros. Esta actividad se realizó completamente a distancia y se concluyó satisfactoriamente, observándose que en breve tiempo los estudiantes adquirieron habilidades en el uso del entorno virtual.

- Construcción del marco teórico. La actividad estuvo dirigida a la construcción del marco teórico del proyecto; durante su desarrollo se observó mucho dinamismo dentro del entorno, el número de participaciones fue bastante alto y cada grupo incrementó sus habilidades en el uso de Synergeia y en la organización de su espacio de trabajo, además, se percibió el cumplimiento de los roles asumidos en la mayoría de los participantes, en los casos contrarios algunos mensajes estuvieron dirigidos a hacer llamados de atención a las personas que no se manifestaban con sus aportes. Finalmente, la actividad concluyó con éxito, tres de los grupos enviaron sus trabajos en la fecha prevista, sólo un grupo lo hizo posteriormente.

- Análisis de la experiencia. Finalmente, la tercera y última actividad se orientó hacia la culminación del proyecto con el análisis de la experiencia venezolana acerca de los entornos virtuales de enseñanza y de aprendizaje en la educación superior, que cada grupo seleccionó en las fases anteriores. El trabajo de los grupos fue muy activo intercambiando y revisando materiales y opiniones acerca del análisis de la experiencia y de la integración del proyecto.

\section{ANÁLISIS DE LOS RESULTADOS OBTENIDOS}

- Logro del aprendizaje basado en proyectos. Para medir el logro del aprendizaje de los estudiantes, se tomaron en cuenta la calidad dela planificación y desarrollo del proyecto; y la participación. De esta manera, los resultados obtenidos de la evaluación final de cada participante, representan el producto de la suma de ambas dimensiones.

- Calidad de la planificación y desarrollo del proyecto. Se utilizó como procedimientola revisión decadaunodelos proyectos alassubdimensiones, indicadores y criterios establecidos:

Planificación de la metodología de trabajo:

- Presenta una introducción donde se contextualiza el trabajo y explica las partes que lo conforman. 
- Presenta índice de contenido y éstos son coherentes con los objetivos planteados.

- Los objetivos específicos son claros y guardan relación con el objetivo general.

- Definición del nivel de Educación Superior, en el cual se enmarca la investigación.

- Descripción de la metodología para desarrollar el proyecto, sobre la base del trabajo colaborativo.

- $\quad$ Especificación de los roles que cumplirá cada participante.

Construcción del marco teórico:

- Presenta antecedentes del tema y son pertinentes para orientar la investigación.

- El desarrollo de los contenidos se presenta de forma organizada.

- Amplitud de contenidos trabajados y actualidad.

- Adecuada definición de términos.

- Las citas son pertinentes al tema tratado.

Análisis de la experiencia:

- Coherencia entre el marco teórico y el análisis.

- Pertinencia con el objetivo general.

- Descripción de la experiencia objeto del análisis.

- Conclusiones acerca del análisis.

- Uso de un sistema único para las referencias bibliográficas.

Presentación de la información:

- La información presentada es clara, precisa, correcta y relevante.

- Los objetivos se presentan redactados con verbos en infinitivos.

- Uso correcto del idioma: corrección gramatical y ortografía.

- Redacción adecuada. 
Los resultados obtenidos del trabajo grupal, arrojaron que la calidad de la planificación y desarrollo del proyecto de tres grupos fue Excelente, y de uno fue Bueno. Esto evidencia que el trabajo colaborativo es muy efectivo para el logro de aprendizajes.

- Participación. Constituyó la evaluación individual, basada en los datos derivados de las intervenciones que efectuó cada estudiante durante la experiencia, de acuerdo con sus respectivas subdimensiones, indicadores y criterios:

- Número de participaciones: se consideraron únicamente las participaciones relacionadas con la actividad correspondiente $(1,2$ ó 3), y que su contenido estuviese dirigido a debatir sobre el tema trabajado en la misma.

- Calidad de las participaciones: sólo se tomaron en cuenta las participaciones que evidenciaron un aporte pedagógico, tales como propuestas de desarrollo de la actividad, aclaratorias, envío de materiales, entre otros.

- Cumplimiento del rol: se observó en las participaciones que evidenciaron el cumplimiento de cada rol.

- Aportes significativos al trabajo: representado por los aportes más significativos como: revisiones de los avances, envío de material procesado.

Los resultados obtenidos de las participaciones individuales evidencian que un $35 \%$ se ubican en la categoría Excelente, y un $35 \%$ en la categoría Bueno, lo cual constituye el 70\% de los estudiantes; un 10 \% Regular, y un 20\% Deficiente. Puede afirmarse entonces que un último porcentaje (70\%) logró los objetivos de manera Buena y Excelente.

Es conveniente destacar que los resultados obtenidos del trabajo grupal, se ubicaron en las categorías Excelente y Bueno en un 100\%, mientras que los del trabajo individual el 70 \% se ubicó en las categorías Excelente y Bueno. Estos resultados también evidencian la efectividad del trabajo colaborativo.

Los resultados obtenidos de la evaluación total de cada participante, demostraron que el $40 \%$ de los estudiantes se ubica en la categoría Excelente, y un 35\% de los estudiantes se ubica en la categoría Bueno, lo que evidencia que en un alto porcentaje 
(75\%) el logro del aprendizaje basado en proyectos fue muy efectivo; el 15\% se ubica en la categoría Regular, lo que demuestra que en ese grupo de alumnos el logro del aprendizaje basado en proyectos fue medianamente efectivo; y sólo el 10\% se ubica en la categoría Deficiente; en ellos el logro del aprendizaje basado en proyectos fue nada efectivo.

\section{- Percepción sobre la importancia del trabajo colaborativo}

Para medir esta variable, se consideraron las dimensiones valor social, valor personal y valor educativo, y sus respectivos indicadores y criterios:

\section{Valor Social}

- El trabajo colaborativo ayuda a trabajar de un modo eficaz en grupo, mejorando su integración y cohesión.

- Mejora la socialización y las relaciones interpersonales.

- $\quad$ Aumenta la tolerancia respecto a los integrantes del grupo, y a las ideas que se han establecido dentro del mismo.

- Ayuda a integrar a los alumnos con más dificultades al favorecer el progreso del grupo y de cada uno de sus componentes.

Valor personal

- Aumenta la autoestima y la valoración personal.

- Aumenta el control individual y favorece las conductas reflexivas.

- Favorece el aprendizaje de habilidades y conductas propias que ayudan a elaborar pautas de comportamiento para etapas vitales futuras.

- $\quad$ Ayuda a desarrollar actitudes más positivas hacia los otros, como el respeto y valoración, confianza y colaboración, solidaridad y empatía.

- $\quad$ Representa un alto poder motivador para los alumnos.

Valor Educativo

- Mejora la productividad y el rendimiento académico.

- Potencia una comunicación más eficaz y satisfactoria, con un lenguaje más elaborado, de mayor precisión y rigor, en los intercambios personales y grupales. 
- Aumenta la participación del estudiante.

- $\quad$ Promueve el desarrollo de la responsabilidad frente a los demás y frente a su propio aprendizaje.

- Aumenta las actitudes que los alumnos tienen hacia los contenidos, potenciando el interés y la motivación intrínseca hacia el aprendizaje.

- Potencia el aprendizaje de actitudes (responsabilidad, colaboración $\mathrm{y}$ respeto) y la adquisición de habilidades sociales (comunicativas y asertivas).

- Mejora las estrategias específicas con que el alumno se enfrenta a los conocimientos (resolución de problemas, desarrollo del pensamiento divergente y creativo, y capacidad de expresión de las ideas y pensamiento).

El análisis de los resultados se hizo según la opinión de los estudiantes sobre el trabajo colaborativo: a) por total de dimensiones, b) por cada una de las tres dimensiones en particular.

- Percepción sobre la importancia del trabajo colaborativo, por total de dimensiones

Los resultados obtenidos de las opiniones evidencian que un $49.2 \%$ de los estudiantes estuvo Muy de acuerdo, y un 39.4\% en la categoría De acuerdo, lo cual constituye el $89 \%$ de los estudiantes; un $9 \%$ no estuvo Ni de acuerdo ni en desacuerdo, un 2.4\% se manifestó En desacuerdo, y en la categoría Muy en desacuerdo no se obtuvo ninguna opinión. Puede afirmarse entonces que un altísimo porcentaje (89\%) de los estudiantes están Muy de acuerdo y De acuerdo con la importancia del trabajo colaborativo.

- Percepción sobre la importancia del trabajo colaborativo, por dimensión

Los resultados obtenidos de las opiniones acerca del valor social demuestran que un $42.2 \%$ de los estudiantes estuvo Muy de acuerdo, y un 40.6\% se ubicó en la categoría De acuerdo, lo cual constituye el 83\% de los estudiantes; un $15.6 \%$ no estuvo Ni de acuerdo ni en desacuerdo, un 1.6\% se manifestó En desacuerdo, y en la categoría Muy en desacuerdo no se obtuvo ninguna opinión. Puede afirmarse entonces que el (83\%) de los estudiantes opinó estar Muy de acuerdo y De acuerdo, con relación al valor social atribuido al trabajo colaborativo. 
Los resultados obtenidos de las opiniones acerca del valor personal evidencian que un $52.5 \%$ de los estudiantes estuvo Muy de acuerdo, y un $38.8 \%$ estuvo De acuerdo, lo cual constituye el $91 \%$ de los estudiantes; un $6.2 \%$ no estuvo $\mathrm{Ni}$ de acuerdo ni en desacuerdo, un $2.5 \%$ se manifestó En desacuerdo, y en la categoría Muy en desacuerdo no se obtuvo ninguna opinión. Puede afirmarse entonces que el (91\%) de los estudiantes opinó estar Muy de acuerdo y De acuerdo, con el valor personal que se le asigna al trabajo colaborativo.

En cuanto al valor educativo, los resultados obtenidos de las opiniones evidencian que un 50.9\% de los estudiantes estuvo Muy de acuerdo, y un $39.3 \%$ estuvo De acuerdo, lo cual constituye el $90 \%$ de los estudiantes; un $7.1 \%$ no estuvo $\mathrm{Ni}$ de acuerdo ni en desacuerdo, un $2.7 \%$ se manifestó En desacuerdo, y en la categoría Muy en desacuerdo no se obtuvo ninguna opinión. Puede afirmarse entonces que el (90\%) de los estudiantes opinó estar Muy de acuerdo y De acuerdo, con el valor educativo del trabajo colaborativo.

\section{- Habilidades Sociales}

El procedimiento realizado para medir las habilidades sociales, específicamente las a) habilidades de conversación, b) habilidades de aceptación asertiva y c) oposición asertiva, se basó en el análisis de 795 participaciones registradas en el entorno Synergeia y 3 efectuadas a través del correo electrónico, resultando un total de 798 intervenciones. Las tres habilidades mencionadas, se operacionalizaron atendiendo a sus particularidades, en cuanto a la dimensión e indicadores que independientemente presentan, de esta manera se asumió el análisis de cada habilidad o variable por separado.

El primer procedimiento realizado fue la revisión de cada participación a fin de identificar el propósito o temática de cada una, como producto se obtuvo que las 798 intervenciones registradas en Synergeia y en el correo, tuvieron nueve propósitos distintos, a continuación se mencionan:

- Organizar los grupos de trabajo

- Establecer la metodología de trabajo

- Construir el marco teórico del proyecto

- Intercambiar información sobre un tema

- Solicitar ayudas

- Analizar la experiencia de Educación a Distancia 
- Presentar el informe final

- Solicitar ayuda sobre aspectos técnicos

- $\quad$ Sobre otros tópicos

Los resultados obtenidos de acuerdo al número total de participaciones según el propósito evidencian que las participaciones con el propósito de construir el marco teórico del proyecto e intercambiar información sobre un tema, fueron las más frecuentes, con porcentajes superiores al $20 \%$ cada una, y un total de $43 \%$ entre las dos. Luego les siguen las participaciones con el propósito de analizar la experiencia de educación a distancia, con un 19\%, y las participaciones con el propósito de presentar el informe final, con un 11\%, con un total de $30 \%$ entre las dos. Por último, las participaciones menos frecuentes fueron con los propósitos de organizar los grupos de trabajo, establecer la metodología de trabajo, solicitar ayudas, solicitar ayuda sobre aspectos técnicos e intercambiar sobre otros tópicos, con porcentajes iguales o menores al $7 \%$, con un total de $27 \%$ entre todas.

A continuación se analizan las tres variables atendiendo a sus especificidades, el procedimiento llevado a cabo es común para las tres variables, sólo varían las dimensiones e indicadores.

\section{- Habilidades de conversación}

Para medir esta variable, se analizó la presencia, en cada una de las 798 participaciones, de las cinco dimensiones planteadas en la operacionalización de la variable, afecto, preguntas conversacionales, comentarios autoreveladores, componentes de refuerzo o cumplidos y respuestas concretas. A continuación se presentan los indicadores:

- Tono emocional y respuesta apropiada a la interacción y al contenido escrito.

- $\quad$ Preguntas dirigidas al interlocultor para obtener información sobre él.

- Información suministrada al interlocutor sobre los intereses, aficiones personales.

- Comentarios, expresiones o preguntas para expresar halago, aprobación o comprensión del mensaje.

- $\quad$ Respuestas claras y concisas. 
Los resultados arrojaron que el 95\% de las intervenciones evidencian que los estudiantes poseen un grado Muy alto y Alto de habilidades sociales de conversación, mientras que el $5 \%$ restante se ubica en las categorías de Regular, Poco y Ninguno.

\section{- Habilidades de aceptación asertiva}

Igual que para la variable anterior se analizó en este caso, la presencia de las cuatro dimensiones establecidas en la operacionalización, en las 798 participaciones. Las dimensiones son: afecto, expresión de sentimientos personales, expresiones de aprecio y elogio, conducta positiva recíproca. Los indicadores correspondientes son:

- Tono emocional y respuesta apropiada a la interacción y al contenido escrito.

- Frases que transmitan información al interlocutor acerca de los sentimientos positivos que les ha producido su conducta.

- $\quad$ Declaraciones escritas que indican aprecio o elogio del buen desempeño del interlocutor.

- Ofrecimiento para devolver en el futuro alguna ayuda al interlocutor.

Los resultados demuestran que el $96 \%$ de las intervenciones evidencian que los estudiantes poseen un grado Muy alto y Alto de habilidades sociales de aceptación asertiva, y que sólo el $4 \%$ de las intervenciones de los estudiantes, las presenta en un grado Regular, Poco o Ninguno.

\section{- $\quad$ Habilidades de oposición asertiva}

Las dimensiones trabajadas para esta variable fueron: comprensión de lo que el otro dice o expresión del problema, desacuerdo, petición de un cambio o propuesta de solución. Los indicadores para estas dimensiones son los siguientes:

- $\quad$ Frases que evidencian la comprensión del problema, pero no su acuerdo con la postura del interlocutor. El inicio de la frase debe indicar la naturaleza u origen del conflicto, esto ayuda a suavizar la respuesta y a que no se perciba como agresiva

- Frases que transmiten resistencia, oposición o desacuerdo con el interlocutor. 
- Petición explícita al antagonista para que cambie su conducta inaceptable, o propuesta de solución para la situación conflictiva.

Los resultados demuestran que el $87 \%$ de las intervenciones evidencian que los estudiantes poseen un grado Muy alto y Alto de habilidades sociales de oposición asertiva, mientras que el $13 \%$ restante se ubica en las categorías de Regular, Poco o Ninguno.

\section{DISCUSIÓN DE LOS RESULTADOS}

La discusión se aborda desde tres perspectivas relevantes en el estudio que conducen a determinar las ventajas que pudiera aportar el trabajo colaborativo a través de un entorno virtual para promover el desarrollo de habilidades sociales, en el contexto universitario.

Los resultados de la primera actividad, la cual dio inicio a la experiencia de trabajo colaborativo desarrollada durante seis semanas a través del entorno virtual Synergeia, permitieron constatar que las metodologías seleccionadas para llevar a cabo esta tarea fueron adecuadas y constituyeron las bases para alcanzar resultados muy efectivos en el logro de aprendizajes en los estudiantes.

En lo que se refiere a la organización del proyecto, los resultados concuerdan con el modelo de investigación en grupo planteado por Herbert Thelen, completado por Sharan (Román, 2002), en cuanto a la efectividad alcanzada al realizar un plan de organización general de la clase, facilitando a los estudiantes el trabajo en pequeños grupos, utilizando aspectos como la investigación cooperativa, las discusiones grupales y la planificación de proyectos. Además, siguiendo los pasos propuestos para trabajar este modelo, se organizaron las actividades atendiendo a la selección del tópico o tema; la planeación cooperativa de metas, tareas y procedimientos; la implementación; monitoreo del profesor; el análisis y síntesis de lo trabajado y del proceso seguido; la presentación del producto final y la evaluación.

Así mismo, se aplicó el método del Aprendizaje por Proyectos, que en opinión de Pérez (2002) citado por Méndez (2003, p.3), "Los proyectos se forman por un problema o cuestión que dirige y organiza las actividades de aprendizajes, produciendo diferentes resultados que de forma acumulativa dan lugar a un producto final con relación a la cuestión planteada". De esta manera, el proyecto realizado por los estudiantes funcionó como el eje central de investigación a través del cual giraron 
todas las actividades de trabajo colaborativo y las interacciones producidas en el entorno virtual.

Se encontró que al tomar en cuenta algunas sugerencias del método del Aprendizaje por Proyectos para organizar por etapas la entrega de productos estableciendo, tres momentos relacionados con: la planificación de la metodología de trabajo, la construcción del marco teórico y el análisis de la experiencia de educación a distancia, se facilitó a los estudiantes el tratamiento de la información, el establecimiento de sus propias relaciones entre los diferentes contenidos, y la construcción de su propio conocimiento; esto también concuerda con los planteamientos de Jorrín y otros (s/f), en cuanto a las ventajas de este método.

La organización del trabajo fundamentada en las metodologías antes mencionadas, permitieron observar durante y después del proceso todas las intervenciones producidas durante las seis semanas, lo que dio lugar a evaluar el desempeño individual de cada estudiante considerando las etapas o momentos relacionados con el proyecto, obteniendo como resultado que para un alto porcentaje, el $70 \%$ de los estudiantes, el logro del aprendizaje basado en proyectos fue muy efectivo.

Igualmente se logró un producto final por grupo, el cual sirvió de insumo para realizar la evaluación grupal, obteniéndose que la calidad de la planificación y desarrollo del proyecto de tres grupos fue Excelente, y de uno fue Bueno. Esto evidencia que el trabajo colaborativo es muy efectivo para el logro de aprendizajes.

En resumen, los resultados obtenidos del trabajo grupal, se ubicaron en las categorías Excelente y Bueno en un 100\%, mientras que en los del trabajo individual el 70 \% se ubicó en las categorías Excelente y Bueno.

A partir de estos resultados, se puede afirmar que el aprendizaje logrado por los estudiantes valorando la importancia de la aplicación de una metodología de trabajo colaborativo basado en el Aprendizaje por Proyectos fue muy efectivo, lo que demuestra que la selección de ambos métodos y la organización del proyecto de acuerdo con las recomendaciones que se ofrecen para estructurarlo se realizó de manera adecuada, arrojando resultados muy efectivos para el logro de aprendizajes en los estudiantes; además se puede inferir la factibilidad de aplicar estas metodologías a distancia, utilizando entornos virtuales adecuados que respondan a las necesidades de cada situación. 
La segunda actividad orientada a identificar la opinión de los estudiantes acerca de la importancia del trabajo colaborativo, tuvo como base la aplicación de un cuestionario fundamentado en los aportes de Alfageme (2002), en los cuales sintetiza el valor añadido del trabajo colaborativo, en tres grandes aspectos: valor social, valor personal y valor educativo, y de otros autores entre ellos Cabero (2003), quien destaca elementos similares atribuidos al aprendizaje colaborativo, como el aumento de las destrezas de comunicación y expresión oral, el rol social del aprendizaje como producto de la interacción entre las personas, tanto de forma individual y colectiva como con los materiales de enseñanza y los ambientes donde el aprendizaje se produce.

De esta manera una vez analizada la información con relación a la percepción sobre la importancia del trabajo colaborativo, por el total de las dimensiones, se obtuvo que un altísimo porcentaje, el 89\% de los estudiantes, estuvieron Muy de acuerdo y De acuerdo con la importancia del mismo.

$\mathrm{Al}$ intentar profundizar en cada una de las dimensiones estudiadas, se obtuvo que el 83\% de los estudiantes opinó estar Muy de acuerdo y De acuerdo con el valor social o grado de significación que representa cada individuo dentro del grupo, lo que evidencia que la percepción acerca de la importancia del trabajo colaborativo es favorable en esta dimensión. El 91\% opinó estar Muy de acuerdo y De acuerdo, con el valor personal o grado de significación del individuo hacia sí mismo, lo que demuestra que la percepción acerca de la importancia del trabajo colaborativo es más favorable en esta dimensión. Finalmente, el 90\% de los estudiantes opinó estar Muy de acuerdo y De acuerdo, con el valor educativo o el interés y disposición del individuo hacia su propio aprendizaje, lo que evidencia que la percepción acerca de la importancia del trabajo colaborativo también es favorable en esta dimensión.

Estos resultados muy favorables con relación al valor personal y favorables en cuanto a los valores social y educativo, demuestran que existen concordancias entre la opinión de los estudiantes acerca de la importancia del trabajo colaborativo y los aportes de Alfageme (2002) y Cabero (2003) seleccionados para la elaboración del cuestionario. Además, considerando el altísimo porcentaje obtenido en cuanto al total de dimensiones, se puede afirmar que los estudiantes atribuyen mucha importancia al trabajo colaborativo, por lo tanto este tipo de método pudiese ser aplicable en cualquier situación de enseñanza y de aprendizaje facilitando procesos cognitivos, motivacionales y afectivo-relacionales, según lo afirma Echeita (1995) citado por Díaz (2002). 
La tercera y última actividad dirigida a caracterizar las habilidades de asertividad (aceptación asertiva y oposición asertiva) y de conversación que tuvieron los estudiantes a través del entorno virtual utilizado, se realizó a partir del análisis de todas las participaciones producidas durante las seis semanas de intervención.

Con relación a las habilidades sociales de conversación, los resultados arrojaron que el $95 \%$ de las intervenciones evidencian que los estudiantes poseen un grado Muy alto y Alto de este tipo de habilidades, es decir, demostraron la capacidad de iniciar y mantener conversaciones informales a través de Synergeia, esto supone la coincidencia con los planteamientos de Kelly (1992), quien afirma que de alguna manera disponer de un repertorio conversacional efectivo no sólo permite al individuo salir airoso en las interacciones informales, sino que además es un precursor probable del desarrollo de relaciones duraderas, dado que la formación de tales relaciones requiere, en primer lugar, que el individuo interactúe satisfactoriamente con los demás durante una conversación. Igualmente, los cuantiosos resultados ubicados en un grado muy alto y alto demuestran que los estudiantes a través de sus intervenciones manifestaron la presencia de los componentes conductuales asociados a esta habilidad.

En lo que se refiere a las habilidades sociales de aceptación asertiva, los resultados demuestran que el 96\% de los estudiantes poseen un grado Muy alto y Alto de estas habilidades, debido a que en sus intervenciones pusieron de manifiesto, según lo afirmado por Kelly (1992, p.175) “...la capacidad de un individuo para trasmitir a otra persona sus posturas, opiniones, creencias o sentimientos de manera eficaz y sin sentirse incómodo". Así mismo, estos resultados concuerdan con los componentes conductuales relacionados con esta habilidad, ya que en las participaciones se evidenció en alto grado de respuestas con un tono emocional adecuado y respuesta apropiada a la interacción y al contenido escrito; también, se encontraron frases que transmitieron información al interlocutor acerca de los sentimientos positivos que les produjo su conducta; declaraciones escritas que indicaron aprecio o elogio del buen desempeño del interlocutor; y ofrecimiento para devolver en el futuro alguna ayuda al interlocutor.

Finalmente, se encontró que el $87 \%$ de los estudiantes poseen un grado Muy alto y Alto de habilidades sociales de oposición asertiva, pues se constató la presencia de los componentes conductuales inherentes a esas habilidades, es decir, se encontraron frases que denotaron la comprensión de lo que el otro dice o expresión del problema, desacuerdo y petición de un cambio o propuesta de solución, situación que concuerda con su objetivo, el cual, parafraseando a Kelly (1992), no es descargar comentarios 
beligerantes sobre el otro, sino es el de comunicar tranquilamente los sentimientos e invitar al antagonista a que cambie su conducta.

Para resumir, los resultados totales obtenidos para las habilidades sociales de conversación muestran que el $95 \%$ de las intervenciones de los estudiantes poseen un grado Muy alto y Alto de este tipo de habilidades; el $96 \%$ de los estudiantes poseen un grado Muy alto y Alto de habilidades de aceptación asertiva; y un 87\% poseen un grado Muy alto y Alto de habilidades de oposición asertiva, por lo que se puede inferir que en un alto grado los estudiantes demostraron a través del entorno virtual Synergeia, una conducta socialmente habilidosa, lo cual coincide con la opinión de Caballo (1993) citado por Alfageme (2002), como el conjunto de conductas emitidas por un individuo en un contexto interpersonal que expresa los sentimientos, actitudes, deseos, opiniones o derechos de ese individuo de un modo adecuado a la situación y que generalmente resuelve los problemas inmediatos de la situación mientras minimiza la probabilidad de futuros problemas.

En síntesis, se puede afirmar que los resultados obtenidos en cuanto al logro del aprendizaje basado en proyectos fue muy efectivo, lo que demuestra que la selección de ambos métodos y la organización del proyecto de acuerdo con las recomendaciones que se ofrecen para estructurarlo se realizó de manera adecuada, además se puede inferir la factibilidad de aplicar estas metodologías en la modalidad a distancia; con relación a la percepción de los estudiantes sobre el trabajo colaborativo, los resultados fueron muy favorables con relación al valor educativo y favorables en cuanto a los valores personal y social. Además, considerando el altísimo porcentaje obtenido en cuanto al total de dimensiones, se puede afirmar que los estudiantes atribuyen mucha importancia al trabajo colaborativo, por lo tanto este tipo de método pudiese ser aplicable en cualquier situación de enseñanza y de aprendizaje facilitando procesos cognitivos, motivacionales y afectivo-relacionales; y por último, en un alto grado los estudiantes demostraron a través del entorno virtual Synergeia, una conducta socialmente habilidosa en los aspectos conversacionales y de asertividad, donde manifestaron sus sentimientos, actitudes, deseos, opiniones o derechos de un modo adecuado a la situación, por lo que también se puede afirmar que el trabajo colaborativo basado en el Aprendizaje por Proyectos y mediado a través de un entorno virtual en el contexto universitario, promueve el desarrollo de habilidades sociales en los estudiantes. 


\section{CONCLUSIONES Y RECOMENDACIONES}

A partir de los objetivos propuestos y alcanzados mediante la investigación y de los resultados obtenidos, este estudio ha generado algunas conclusiones, aportes y recomendaciones, que a continuación se presentan:

- El aprendizaje logrado por los estudiantes valorando la importancia de la aplicación de una metodología de trabajo colaborativo basado en el aprendizaje por proyectos fue muy efectivo, lo que demuestra que las metodologías seleccionadas en este caso, el modelo de investigación en grupo planteado por Herbert Thelen, completado por Sharan (Román, 2002) y el método del Aprendizaje por Proyectos, resultaron muy adecuadas y constituyeron las bases para alcanzar resultados muy efectivos en el logro de aprendizajes en los estudiantes.

- La aplicación de los métodos de trabajo colaborativo de investigación en grupo y del Aprendizaje por Proyectos son factibles de implementar totalmente a distancia, siempre que se organicen las actividades a partir de los lineamientos que ofrecen estos métodos y se seleccione adecuadamente el entorno virtual que más se adapte a las necesidades de la situación.

- El trabajo colaborativo mediado a través de un entorno virtual en el contexto universitario, ofrece algunas ventajas, entre ellas: aumenta las destrezas de comunicación a través de la expresión escrita, aumenta el rendimiento académico, mejora las habilidades sociales, se incrementa el rol social del aprendizaje como producto de la interacción entre las personas, tanto de forma individual y colectiva como con los materiales de enseñanza y los ambientes donde el aprendizaje se produce.

- Los entornos virtuales de enseñanza aprendizaje son factibles de utilizar y permiten generar espacios educativos completamente a distancia sin la necesidad de encuentros presenciales, sin embargo es necesario considerar la formación y las actitudes que tenga el docente en torno al dominio tecnológico mínimo para desenvolverse eficientemente en estos espacios y sobre todo determinar las estrategias idóneas y los materiales adecuados para trabajar en entornos virtuales.

- La selección del medio o entorno virtual es determinante para obtener resultados satisfactorios, debe primar la flexibilidad y la facilidad de uso 
antes que otros aspectos de forma, el docente está llamado a seleccionar de acuerdo a las características de la audiencia y de los objetivos que se pretendan alcanzar, espacios accesibles de dominio común y fáciles de usar por toda la comunidad.

- Con relación a la percepción de los estudiantes acerca de la importancia del trabajo colaborativo los resultados fueron muy favorables con relación al valor personal y favorables en cuanto a los valores social y educativo, en general se puede afirmar que los estudiantes atribuyen mucha importancia al trabajo colaborativo, por lo tanto este tipo de método pudiese ser aplicable en cualquier situación de enseñanza y de aprendizaje facilitando procesos cognitivos, motivacionales y afectivo-relacionales.

- En cuanto a las habilidades sociales estudiadas se encontró que en un alto grado los estudiantes demostraron a través del entorno virtual Synergeia, una conducta socialmente habilidosa en los aspectos conversacionales y de aceptación y oposición asertiva, manifestando sus sentimientos, actitudes, deseos, opiniones o derechos de un modo adecuado a cada situación.

- Finalmente, los resultados de esta investigación permitieron comprobar que aplicar una metodología de trabajo colaborativo basada en el Aprendizaje por Proyectos y mediada a través de un entorno virtual en el contexto universitario, promueven el desarrollo de habilidades sociales de conversación y de aceptación y oposición asertiva en los estudiantes.

\section{RECOMENDACIONES}

- Profundizar la investigación sobre los métodos de trabajo colaborativo idóneos para desarrollar experiencias de enseñanza y de aprendizaje a través de entornos virtuales.

- Considerar el diseño estrategias instruccionales basadas en métodos de trabajo colaborativo, que garanticen no sólo la efectividad tanto del aprendizaje como del medio, sino también el desarrollo de habilidades sociales.

- Concebir el uso de entornos virtuales de enseñanza aprendizaje considerando la adecuada selección del mismo, de las estrategias idóneas y los materiales adecuados para trabajar en ellos, y la formación y actitudes que tenga el docente 
y los alumnos en cuanto al dominio tecnológico necesario para interactuar con el medio.

- Incentivar el desarrollo de habilidades sociales tanto en los docentes como en los alumnos con el propósito de mejorar posibles problemas en la educación.

- Ampliar el campo de investigación sobre el desarrollo o promoción de las habilidades sociales en entornos virtuales de trabajo colaborativo, a fin de generalizar su uso y generar nuevos conocimientos.

\section{REFERENCIAS BIBLIOGRÁFICAS}

Alfageme, B. (2002). Modelo colaborativo de enseñanza-aprendizaje en situaciones no presenciales: un estudio de caso. Tesis doctoral inédita. España: Universidad de Murcia.

Cabero, J. (2003). Principios pedagógicos, psicológicos y sociológicos del trabajo colaborativo: su proyección en la teleenseñanza. Redes de comunicación en la enseñanza. Barcelona: Paidós Ibérica S.A. Ediciones.

Cebrián, M. (Coord.) (2003). Enseñanza Virtual para la Innovación Universitaria. Madrid: Narcea S. A. de Ediciones.

Gisbert, M.; Adell, J.; Rallo, R.; Bellver, A. (1997). Entornos Virtuales de Enseñanza-Aprendizaje. El Proyecto GET. Cuadernos de Documentación Multimedia. [en línea] No. 6-7. España. Disponible en: http://www.ucm.es/info/ multidoc/multidoc/revista/cuad6-7/ evea.htm [consulta 2006, 28 de enero]

Hernández, R.; Fernández, C.; Baptista, P. (1998). Metodología de la Investigación. México: McGraw-Hill/Interamericana Editores. 2da. Edición.

Jorrín, I.; Vega, G.; Gómez, E. (s/f). El papel facilitador de las TIC en un proceso de aprendizaje colaborativo. Universidad de Valladolid. Relatec, Revista Latinoamericana de Tecnología Educativa. [en línea] Vol. 3, $\mathrm{N}^{\mathrm{o}} 1$.
Disponible en: http://158.49.119.99/ crai/personal/relatec/Jorrin.pdf [consulta 2005, 4 de septiembre]

Kelly, J. (1992). Entrenamiento de las habilidades sociales. Bilbao: Biblioteca de Psicología Desclée de Brouwer. 3ra. Edición.

Méndez, P. (2003). El trabajo pedagógico colaborativo en la educación superior, mediado por herramientas tecnológicas. Ponencia presentada en EDUTEC 2003. Caracas.

Prendes, M. (2003). Aprendemos. ¿cooperando o colaborando?. Las claves del método, en: Martínez S. F. (comp.) (2003). Redes de comunicación en la enseñanza. Barcelona: Paidós Ibérica S.A. Ediciones.

Román, P. (2002). El trabajo colaborativo mediante redes, en: Aguaded, J. I. y Cabero, J. (2002). Educar en Red. Internet como recurso parala educación. Málaga: Ediciones Aljibe.

Zañartu, L. (2003). Aprendizaje colaborativo: una nueva forma de Diálogo Interpersonal en la Red. Contexto Educativo. Revista digital de educación y nuevas tecnologías. Año V, No. 28. 


\section{PERFIL ACADÉMICO Y PROFESIONAL DE LAS AUTORAS}

Nallilda Villasana, profesora de la Universidad Central de Venezuela en el Sistema de Actualización Docente del Profesorado de la Universidad Central de Venezuela (SADPRO-UCV). Licenciatura en Educación, mención Tecnología Educativa y Máster en Educación, mención Tecnologías de la Información y la Comunicación, de la UCV. Profesora de la Maestría en Educación mención Tecnologías de la Información y la Comunicación, UCV.

E-mail: nallilda@yahoo.es

DIRECCIÓN DE LA AUTORA

Calle México, entre $4^{\mathrm{a}}$ y $5^{\mathrm{a}}$ Avenida, $\mathrm{N}^{\circ} 18-17$.

Catia-Caracas. Venezuela.

Elena Dorrego, profesora titular de la Universidad Central de Venezuela, Arquitecto(UCV), Licenciada en Educación(UCV), Máster en Educación(Universidad de Gales, Gran Bretaña), Doctora en Educación (Universidad Nacional Experimental, Simón Rodríguez, Venezuela). Coordinadora de la Maestría en Educación mención Tecnologías de la Información y la Comunicación, UCV.

E-mail: edorrego@cantv.net

DIRECCIÓN DE LA AUTORA

Calle Ecuador, Edif. Padamo PH, Terraza A del Club Hípico.

Caracas 1080, Venezuela

Fecha de recepción del artículo: 11/06/07

Fecha de aceptación del artículo: 6/09/07 